\title{
SURGICAL MANAGEMENT OF URETEROPELVIC JUNCTION OBSTRUCTION IN ADULTS
}

\author{
SANKAR KAUSIK, JOSEPH W. SEGURA \\ Department of Urology, Mayo Medical School, Mayo Clinic, Rochester, Minnesota, USA
}

\begin{abstract}
Ureteropelvic junction (UPJ) obstruction is a well-recognized entity that may present at any time - in fetal life, infancy, childhood, or early or late adulthood. As the most common site of obstruction in the upper urinary tract, the UPJ is an area with which urologists should be well familiar. There has been an improved understanding of the pathophysiology of primary congenital UPJ obstruction that has been reflected in the evolution of surgical options, from open surgical repair to minimally invasive surgery.

Although the primary scope of this review is the surgical management of this condition, we will briefly review the pathogenesis, clinical presentation, and diagnosis of UPJ obstruction.
\end{abstract}

Key words: kidney; kidney pelvis; ureteral obstruction; surgery; percutaneous

Int Braz J Urol. 2003; 29: 3-10

\section{PATHOPHYSIOLOGY}

Ureteropelvic junction (UPJ) obstruction may be defined as a functional or anatomic obstruction to urine flow from the renal pelvis to the proximal ureter that results in symptoms or renal damage. UPJ obstruction does not represent a single anatomic entity, but rather a group of obstructive processes that result from multiple etiologic factors. Congenital UPJ obstruction most often is a result of an intrinsic process, specifically the presence of an aperistaltic segment of the ureter (1). Histologically the lumens of stenotic UPJs are lined with the usual transitional cell epithelium, but are surrounded by an abnormal longitudinal muscle bundle or fibrous tissue. As a result, patients have functional failure of effective peristalsis and inadequate luminal distension to accommodate urinary bolus. Although extrinsic compression by kinks, bands, polar vessels, and a high insertion of the ureter may be obvious, the primary lesion is intrinsic.

\section{CLINICAL PRESENTATION AND DIAGNOSIS}

Congenital UPJ obstruction can present at any time, from intrauterine life to old age. With the increased use of prenatal ultrasound, a number of infants are found to have hydronephrosis. UPJ obstruction is one of the most common causes of prenatal hydronephrosis.

In adults, the majority present with flank pain that can be associated with nausea. Others may present with hematuria, urinary tract infections, stone disease, or vague gastrointestinal complaints. Radiographic evidence plays a key role in the diagnosis of UPJ obstruction. In our opinion, the best radiographic study is a diuretic intravenous pyelogram (2). Other useful studies include radionuclide renal scans, computed tomography, ultrasonography, and retrograde pyelography. The diagnosis of UPJ obstruction is based on the combination of clinical manifestations, radiographic evidence of obstruction and impairment of renal function. 


\section{SURGICAL MANAGEMENT}

The primary indications for treatment of UPJ obstruction include relief of pain and relief of physiologically significant obstruction. In addition, recurrent stone formation or infection may indicate the need for surgical reconstruction of the UPJ. The ultimate goal is to provide a drainage system with unobstructed urinary flow (3).

Although there are a variety of surgical approaches to correction of UPJ obstruction, they can be classified into 3 categories: 1)- Open surgical procedures - pyeloplasty; 2)- Endoscopic (antegrade or retrograde) procedures; 3)- Laparoscopic procedures. While considering these various options, it is important to weigh the potential risks and benefits of these approaches, the success rates, and to keep in mind that long-term results are pending in some cases.

\section{Open Surgical Repair - General Considerations}

Although a number of incisions for performance of a pyeloplasty have been described, the most popular anatomic approach to the UPJ is the extraperitoneal flank approach (3). When this incision is utilized through the bed of the twelfth rib, it typically provides excellent exposure of the UPJ. An anterior extraperitoneal approach is useful in horseshoe kidneys, or where there is anterior malrotation of the kidney. In addition, it can be utilized in thin patients, or for those who have had prior flank operations. The posterior lumbotomy approach can be considered in cases with a significant extraperitoneal component of the UPJ; however it has never gained popularity in the United States.

As a general rule with the open surgical procedures, loss of functional parenchyma and ligation of renal vessels should be avoided. The cases which involve the aberrant anterior crossing vessels are actually lower pole segmental arteries, and these should be preserved by placing them posterior to the reconstructed UPJ (1).

The routine intraoperative placement of ureteral stents and nephrostomy tubes remain somewhat controversial, and varies among the pediatric and adult practices. In our institution we primarily use ureteral stents to decrease the amount of extravasation, and thus limit the risk of secondary fibrosis. External drainage of the operative field is crucial as it prevents urinoma formation, secondary fibrosis, and scarring.

\section{Dismembered Pyeloplasty}

This procedure was popularized and modified by Anderson \& Hynes (4), and can be easily applied or modified to reconstruct the vast majority of UPJ obstructions. It is this versatility that makes it the most popular of all open procedures. When compared to the flap procedures, only a dismembered pyeloplasty allows the excision of the anatomically strictured area. In addition, its utilization is not dependent on whether the ureteral insertion is high or normal. One of the few scenarios where the dismembered pyeloplasty does not provide a good result is when there is a lengthy proximal ureteral stricture associated with a poorly accessible intrarenal pelvis.

After exposing the proximal ureter and renal pelvis to identify the UPJ obstruction, care must be taken to handle the periureteral tissue as atraumatically as possible. This is important in preserving the delicate ureteral vasculature. Fine marking sutures should be placed on the medial and lateral aspects of the renal pelvis, just superior to the UPJ obstruction, and on the lateral aspect of the ureter, inferior to the area that is to be transected. This will maintain proper orientation. The UPJ area is then excised and the lateral aspect of the ureter is spatulated. The superior aspect of the renal pelvis is closed to its most dependent aspect where the ureteral anastomosis is performed. The apex of the spatulated ureter is then anastomosed to the most inferior aspect of the renal pelvis, while the medial portion of the ureter is sutured to the superior aspect of the newly constructed UPJ. The anastomosis should be performed with absorbable sutures placed full thickness through the ureteral wall and renal pelvis, in an interrupted or running fashion (Figure-1).

\section{Culp-DeWeerd Spiral Flap}

Although only occasionally used because of the ease and success of the dismembered pyeloplasty, 

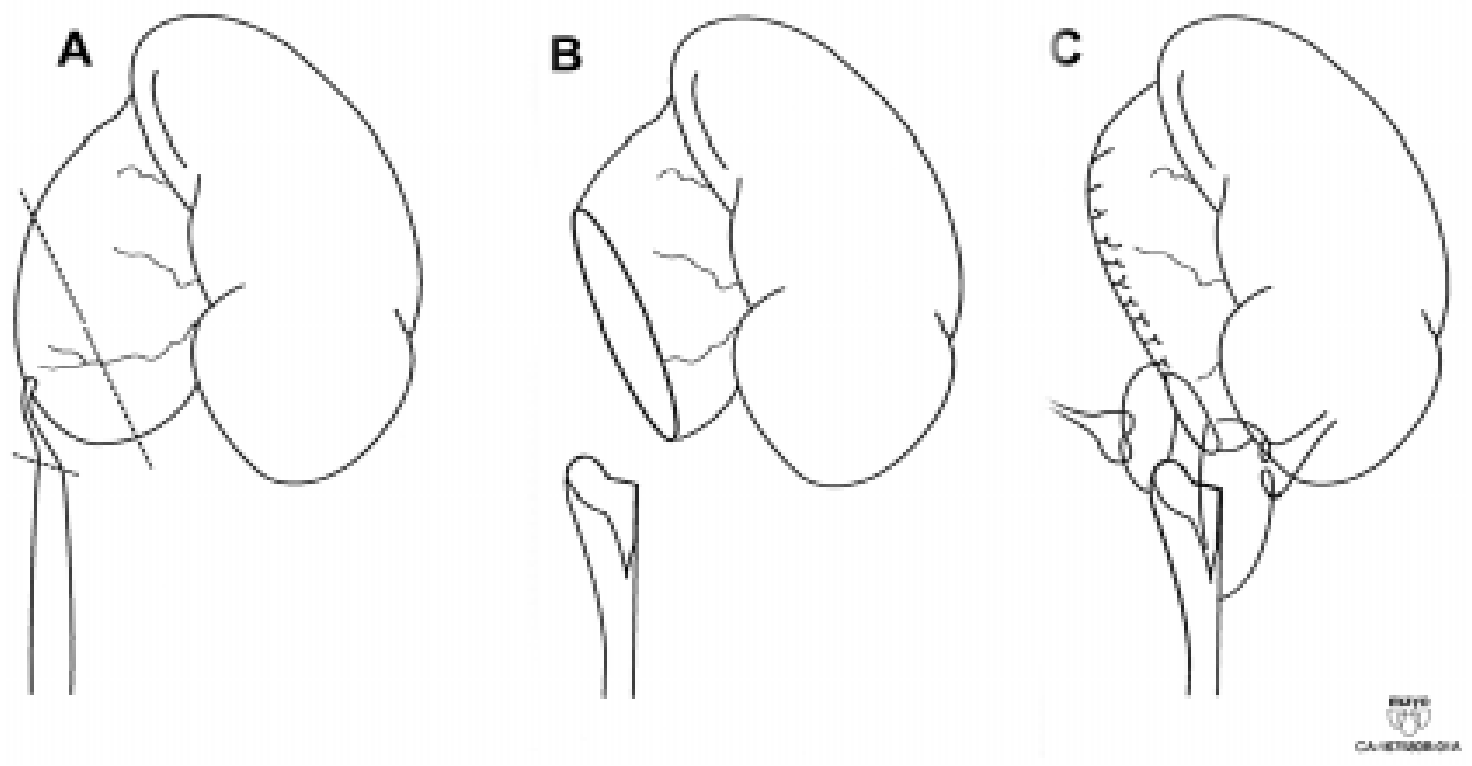

Figure 1 - Anderson-Hynes pyeloplasty.

this spiral flap has its utility (5). The primary role of this procedure is when there is a proximal ureteral stricture associated with a UPJ obstruction. To be effective, the spiral flap should be performed in the presence of a large extrarenal pelvis, as the size of the flap is limited only by the renal pelvis. UPJ obstruction associated with high insertion of the ureter can be difficult to repair with this technique. Figure2 illustrates the procedure.

\section{Foley Y-V Plasty}

This procedure has also been supplanted by the dismembered pyeloplasty. It was originally developed to reconstruct the obstructed system associated with a high ureteral insertion into the renal pelvis. It is not well suited when a proximal ureteral stricture is present, where lower pole vessel
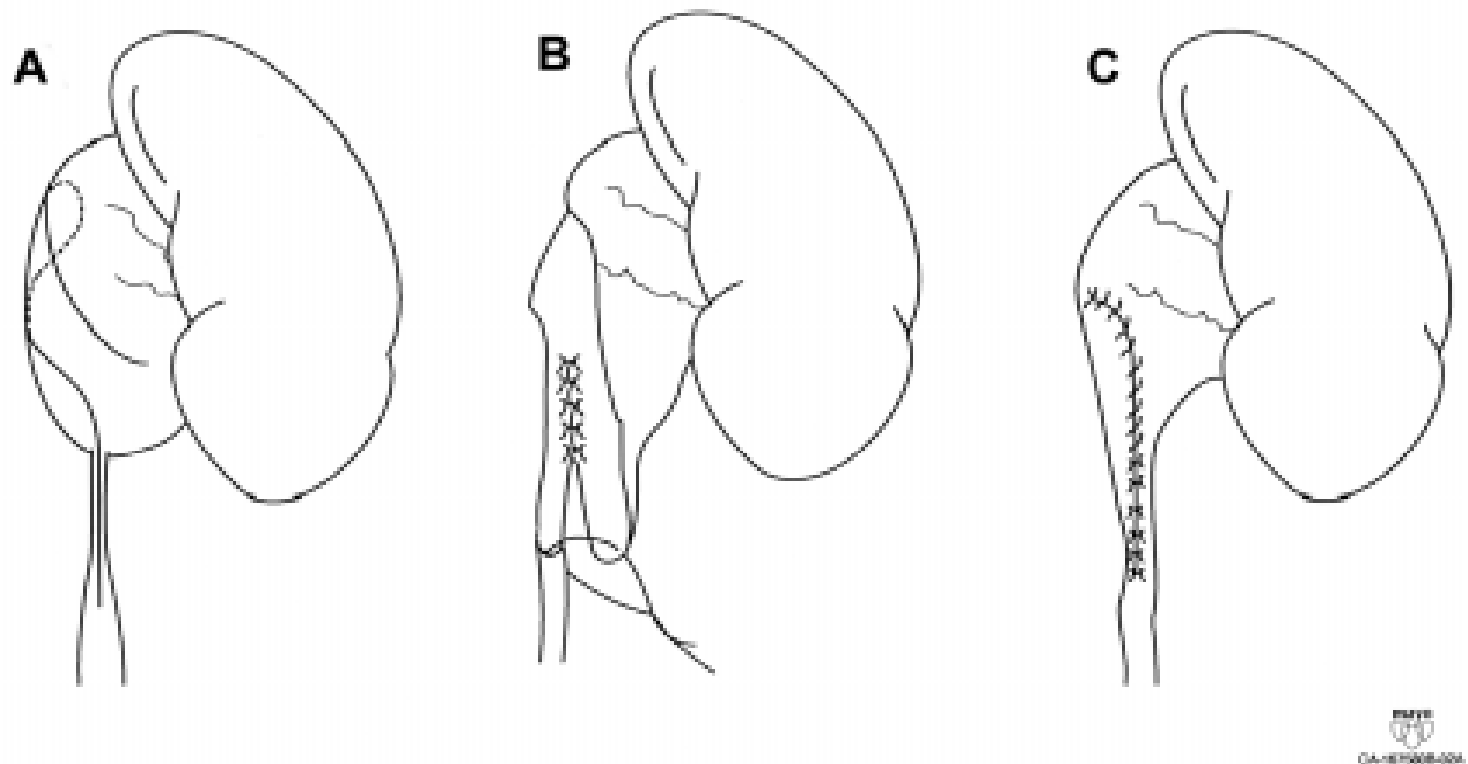

Figure 2 - Culp-DeWeerd spiral flap. 

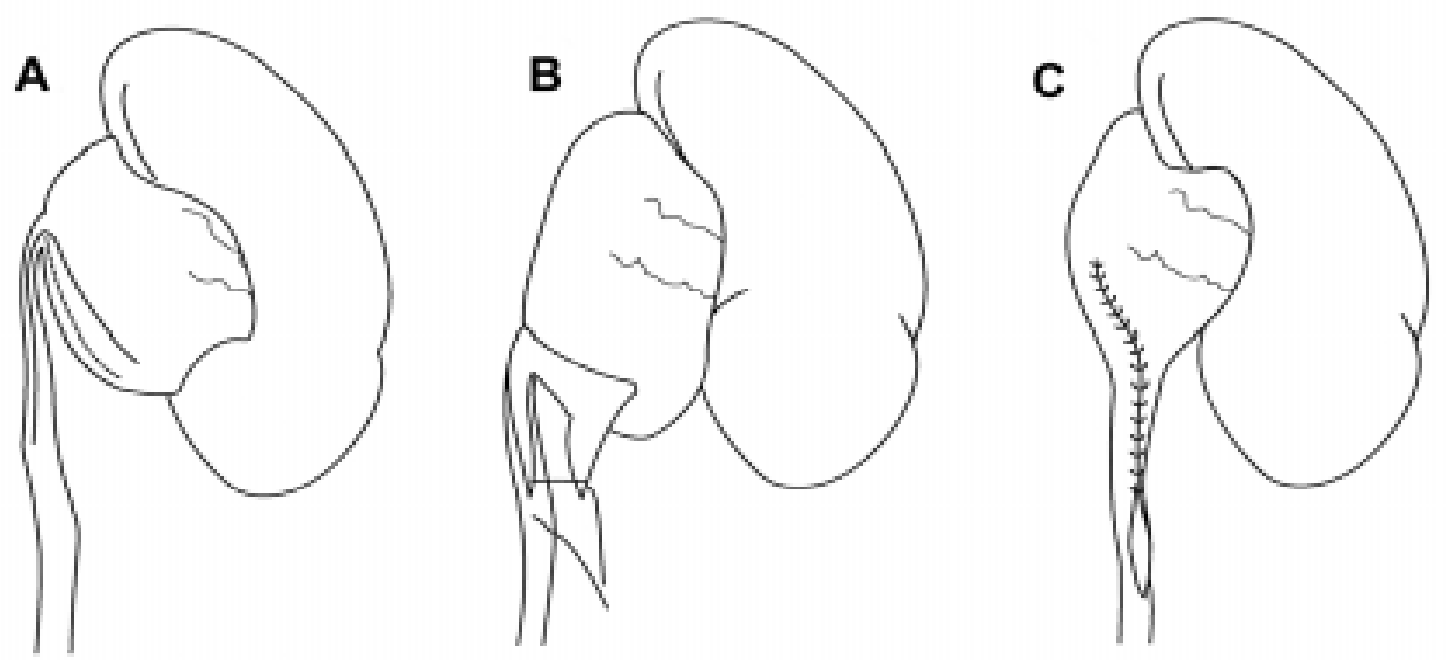

Figure 3 - Foley Y-plasty.

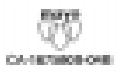

transposition is indicated, or when the reduction of the renal pelvis is desirable. Figure-3 illustrates the procedure.

\section{Scardino-Prince Vertical Flap}

This flap as described by Scardino \& Prince is largely of historic interest only (6). Its application was limited to obstruction of an already dependent UPJ that was situated on the medial aspect of an extrarenal pelvis (Figure-4). Although the vertical flap can be used to manage proximal ureteral strictures, it cannot provide the length and versatility of the spiral flap.

\section{Ureterocalycostomy}

Ureterocalycostomy is an important procedure in certain clinical situations. It is most commonly employed as a salvage procedure after a
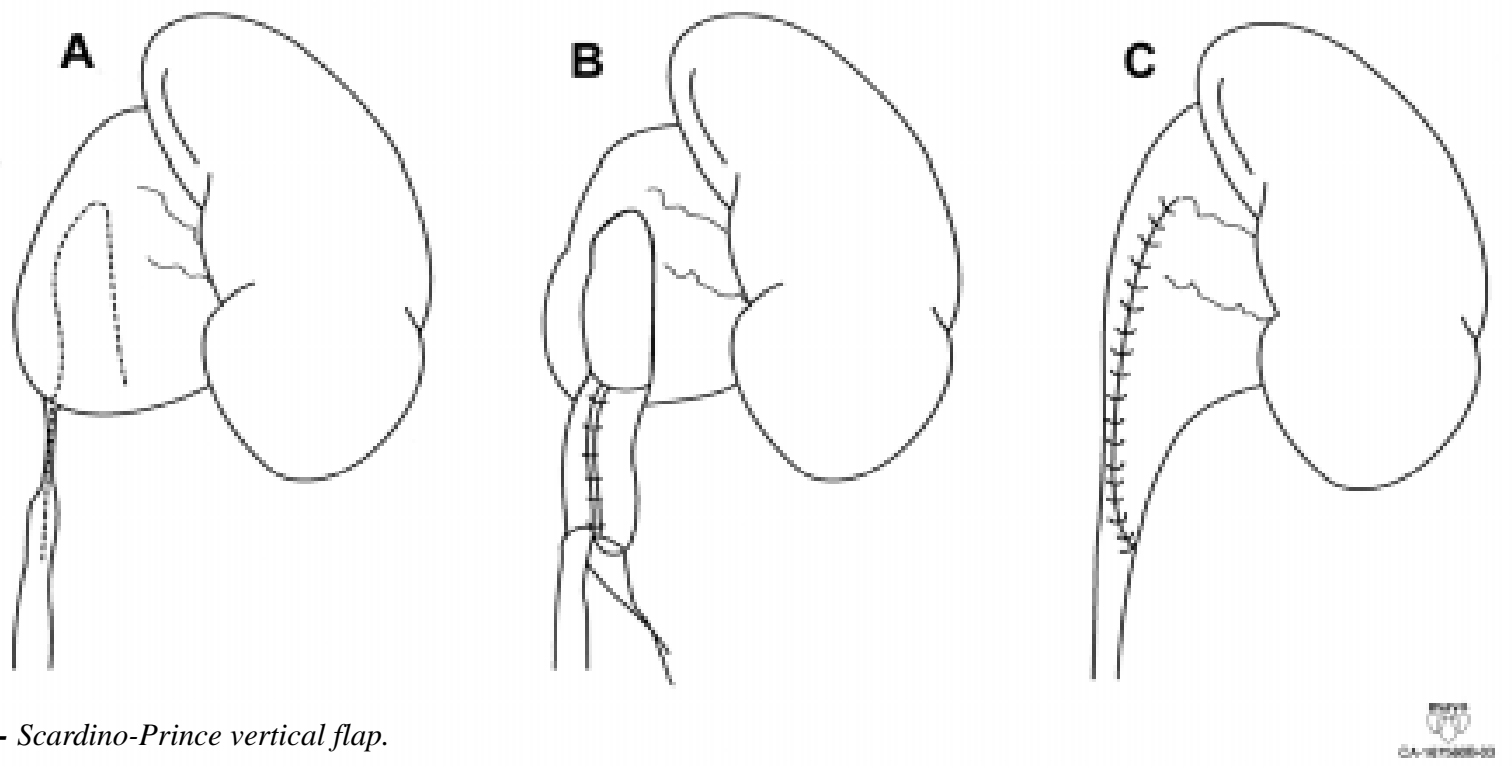

Figure 4 - Scardino-Prince vertical flap. 
A

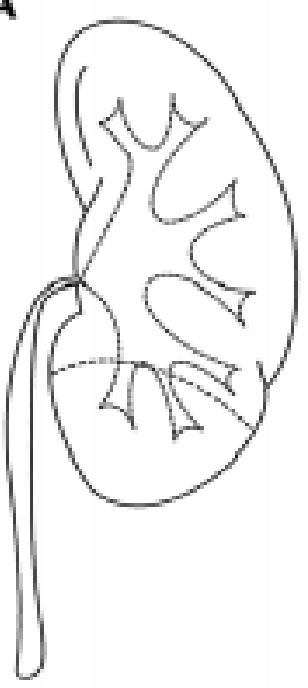

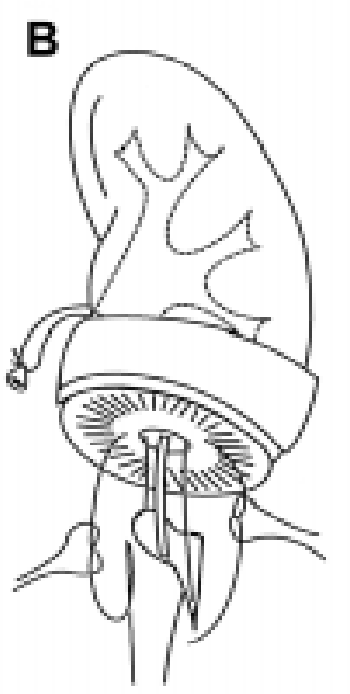

Figure 5 - Complex ureterocalycostomy.

failed pyeloplasty, particularly in situations where a repeat pyeloplasty will likely fail secondary to fibrosis of the renal pelvis. Ureterocalycostomy may also be used as the primary reconstructive procedure for UPJ obstructions associated with rotational or fusion anomalies, such as a horseshoe kidney. Indeed, ureterocalycostomy in the presence of a horseshoe kidney allows for dependent drainage of the unit without the need to sacrifice the isthmus. In addition, it can be utilized when a small intrarenal pelvis is present.

Ureterocalycostomy is performed by mobilizing the kidney to allow easy access to the lower pole. The critical portion is to resect sufficient lower pole parenchyma to prevent subsequent renal cortical fibrosis. The inferior calyx is mobilized and then anastomosed to the ureter that has been spatulated laterally. This is demonstrated in Figure-5.

\section{Endoscopic Management of UPJ Obstruction}

Although open dismembered pyeloplasty remains the gold standard for repair of UPJ obstruction, with success rates between $90-95 \%$, with the advent of endourologic equipment and techniques there are several minimally invasive techniques that are applicable to managing UPJ obstruction. Endopyelotomy has its roots dating back to the technique of intubated ureterotomy, which was popularized by Davis $(7,8)$. Now it has become increasingly well accepted for optimal management of primary UPJ obstruction, with success rates that approach open pyeloplasty with significantly lower morbidity. The 2 approaches to endopyelotomy are the antegrade and the retrograde techniques, which will be reviewed further.

\section{Antegrade Endopyelotomy}

As urologists gained experience with percutaneous management of stones in the early 1980s, it became apparent that the same techniques could be applied for the management of UPJ obstruction. Antegrade endopyelotomy, as it is performed in the Unites States, was largely pioneered and refined by Motola et al. (9). Although the initial success rates were not as good as those of the open surgery, with increasing experience and advances in equipment that has now changed, at our institution, antegrade endopyelotomy has become a first-choice 
procedure for the management of UPJ obstruction (10).

The initial step is to gain percutaneous access through a lateral or upper pole calyx, with the patient in prone position. The key step is the successful placement of a guidewire across the UPJ and into the bladder. If this is not possible in an antegrade fashion, it should be performed in a retrograde manner, and then exchanged. A second safety wire is also useful, so as not to lose access to the UPJ. We generally use a Lunderquist-Ring 0.038 torque wire as out working wire. Since the endopyelotomy is performed with this wire, care should be taken to prevent its kinking or bending. The tract is then dilated and a nephroscope is placed. After careful inspection of the collecting system, the cutting instrument is introduced over the wire (Figure-6). Although the endopyelotomy incision was previously made in a posterolateral location, because of the detailed anatomic findings described by Sampaio \& Favorito (11) we now make our incision in the lateral position. If the UPJ does not appear wide enough to accommodate the knife, it should be balloon-dilated. The instrument of choice at our

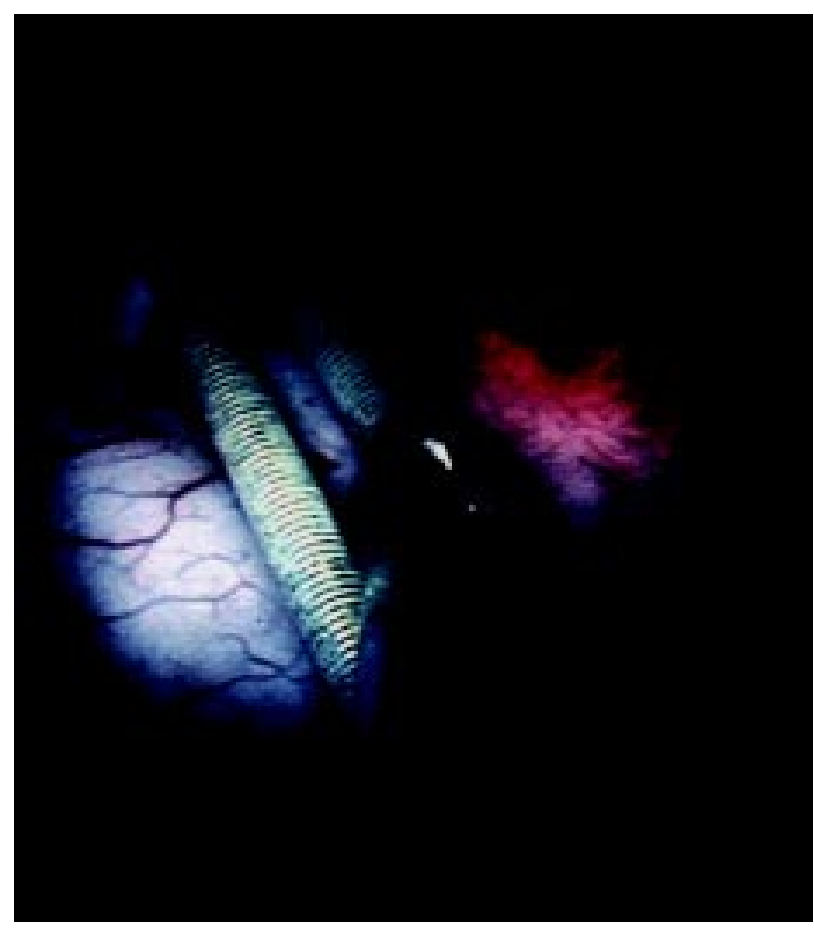

Figure 6 - Introduction of the cold-cutting knife.

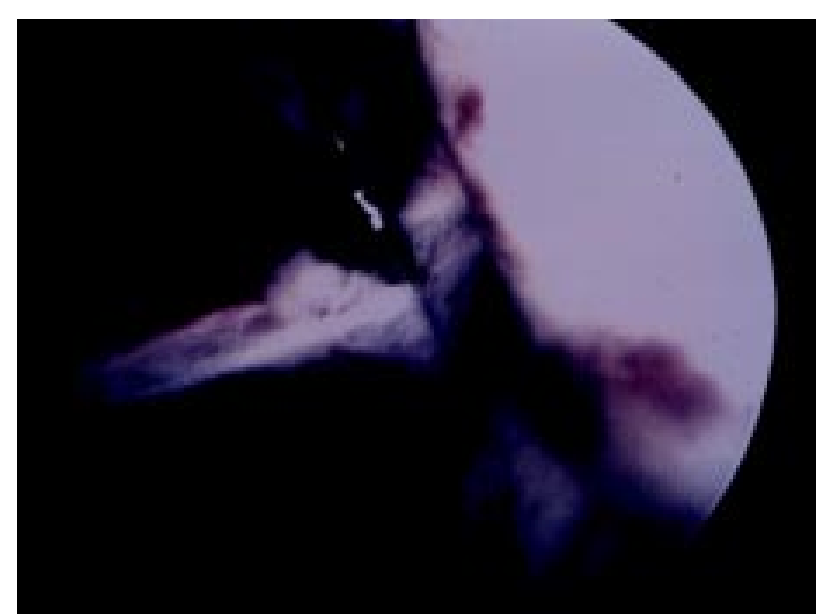

Figure 7 - Cutting laterally.

institution is a cold-cut knife, and the incision is made through the full thickness of the renal pelvis and ureteral wall (Figure-7). This is confirmed by the presence of the wispy retroperitoneal fat identified after the incision. The incision should extend down the ureter at least $1 \mathrm{~cm}$ beyond the area of UPJ obstruction, and should be continued laterally up into the renal pelvis an additional 1 or $2 \mathrm{~cm}$. Care should be taken to avoid any aberrant vessels; these can be identified by their pulsation. If significant bleeding is encountered at the time of the incision, allowing clot formation in the renal pelvis with subsequent vascular tamponade, typically controls the bleeding. If arterial bleeding were to persist despite this, urgent angiography with embolization will be required.

After the incision, a stent should be used and we usually use the $8 \mathrm{~F}$ double-J stent. In addition, we place a $22 \mathrm{~F}$ nephrostomy tube in the renal pelvis. The nephrostomy tube is typically left indwelling for 48 hours, and a trial of clamping is completed prior to its removal. The majority of patients is discharged on post-operative day 2 or 3 , and is able to return to normal activity within a week. Cystoscopic removal of the double-J stents is done at 6 weeks, and an intravenous pyelogram is obtained a few weeks later. All patients are then followed at periodic intervals for signs or symptoms of late failure. We consider the percutaneous endopyelotomy successful if the patient is asymptomatic and the intravenous pyelogram shows improved function or drainage. 


\section{Retrograde Endopyelotomy}

Retrograde management of UPJ obstruction can vary from simple balloon dilation (high failure rate) to the use of Acucise ${ }^{\mathrm{TM}}$ cutting balloon device to ureteroscopic endopyelotomy. A recent Internet survey of over a 1,000 practicing American urologists revealed that Acucise ${ }^{\mathrm{TM}}$ endopyelotomy was the most frequently selected therapy for adults with UPJ obstruction (12). The technique of performing endopyelotomy with the Acucise $^{\mathrm{TM}}$ cutting balloon involves placement of the Acucise $^{\mathrm{TM}}$ catheter over a guidewire across the region of the UPJ. The region of the stricture is noted by the characteristic "waist" seen when inflating the balloon with contrast. The cutting-wire is then positioned laterally and, as the balloon is reinflated, the stricture is simultaneously incised. Extravasation of contrast should be observed, and then an $8 \mathrm{~F}$ double-J stent should be placed. Follow-up is similar to that of antegrade endopyelotomy.

Ureteroscopic endopyelotomy has gained popularity in the treatment of UPJ obstruction (13). Again a wire is passed into the UPJ obstruction, and a rigid or flexible ureteroscope is used to inspect the UPJ and determine the location and length of the narrowed area. An endoscopic incision may then be performed with electrocautery or holmium:YAG laser. The incision is made from just inside the renal pelvis to normal ureter by withdrawing the ureteroscope and the electrode as a unit. The incision is then balloon-dilated to 24F. A ureteral stent should be placed for 6-8 weeks.

\section{Laparoscopic Pyeloplasty}

The first laparoscopic pyeloplasty was performed in 1993 as an alternative to the standard flank pyeloplasty. Details of this procedure have been published recently (14) and are beyond the scope of this review. The principles of laparoscopic pyeloplasty are similar to those of open repair, and dismembered pyeloplasty is the most common approach.

\section{FINAL CONSIDERATIONS}

There are many factors to consider in deciding the optimal surgical procedure to a given patient. Anatomic considerations, past surgical procedures, patient expectations, and the surgeon's experience all contribute to the success of the procedure. The gold standard with success rates of 90-95\% is still the open dismembered pyeloplasty. However, with the trend toward decreasing morbidity and hospitalization, endoscopic management and laparoscopy have come to the forefront. At our institution, the preferred initial management is antegrade endopyelotomy. We have reported an overall success rate of $88 \%$ and this is similar to other published success rates $(9,10,15)$. Although retrograde endopyelotomy is even less invasive, there may be a trade off in terms of success. There have been some comparable results with the retrograde approach; however the follow-up has been inferior. Lastly, laparoscopic pyeloplasty, although technically challenging, also provides early durable results.

\section{REFERENCES}

1. Kletscher BA, Segura JW: Surgical management of UPJ obstruction in adults. AUA Update Series 1996; vol XV: lesson 18.

2. Blute ML, Malek RS: Contemporary concepts in diagnosis and management of UPJ obstruction in adults. AUA Update Series 1990; vol IX: lesson 5.

3. Novick AC, Streem SB: Surgery of the Kidney. In: Walsh PC, Retik AB, Stamey TA, Vaughan Jr ED (eds.), Campbell's Urology. Philadelphia, WB Saunders, 7th ed., 1998.

4. Anderson JC, Hynes W: Retrocaval ureter: A case diagnosed preoperatively and treated successfully by a plastic operation. Br J Urol. 1949; 21:209-14.

5. Culp OS, DeWeerd JH: A pelvic flap operation for certain types of UPJ obstruction: Preliminary report. Mayo Clin Proc. 1951; 26:483-88.

6. Scardino PL, Prince CL: Vertical flap ureteropelvioplasty: Preliminary report. South Med J. 1953; 46:325-31.

7. Davis DM: Intubated ureterotomy: a new operation for ureteral and ureteropelvic strictures. Surg Gynec Obst., 1943; 76:513.

8. Davis DM, Strong GH, Drake WM: Intubated ureterotomy: Experimental work and clinical results. J Urol. 1948; 59:851-62.

9. Motola JA, Badlani GH, Smith AD: Results of 212 
consecutive endopyelotomies: An 8-year follow-up. J Urol. 1993;149:453-6.

10. Kletscher BA, Segura JW, LeRoy AJ, Patterson DE: Percutaneous antegrade endoscopic pyelotomy: Review of 50 consecutive cases. J Urol. 1995; 153, 701-703.

11. Sampaio FJB, Favorito LA: Ureteropelvic junction stenosis: vascular anatomical background for endopyelotomy. J Urol. 1993; 150:1787-91.

12. Hollowell C, Patel R, Bales GT, Gerber GS: Internet and postal survey of endourologic practice patterns among American urologists. J Urol. 2000; 163:177982.

13. Tawhiek ER, Liu J, Bagley DH: Ureteroscopic treatment of UPJ obstruction. J Urol. 1998; 160:1643-7.

14. Jarrett TW: Technique of laparoscopic pyeloplasty. Int Braz J Urol. 2000; 26:76-81.

15. Shalhav AL, Giusti G; Elbahnasy AM, Hoenig DM, Mcdougall EM, Smith DS, et al.: Adult Endopyelotomy: Impact of etiology and antegrade versus retrograde approach on outcome. J Urol. 1998; 160:685-9.

\section{Correspondence address:}

Dr. Joseph W. Segura

Department of Urology, Mayo Clinic

200 First Street SW

Rochester, Minnesota 55905, USA

Fax: + 1507 284-4987

E-mail: segura.joseph@mayo.edu 